\title{
Interacting video information via speech watermarking for mobile second screen in Android smartphone
}

\begin{abstract}
Video identification as an interesting App in Android smartphone has three main aims including: user friendly, identification accuracy and copyright protection. This paper proposes a technique to meet the aims by applying Second Screen Technique. For this reason, the video information is embedded and transmitted within the audio part, and is extracted at destination. The experimental results show that the recognition rate of new technique is very high if no serious noise contaminates the recorded signal. It can be integrated to other mobile communication systems. Moreover, watermarking could be applied for sender/receiver during interaction for improving the security. By marketing this app, selling and advertising revenue increases $30 \%$.
\end{abstract}

Keyword: Video identification; Android smartphone apps; Speech watermarking apps; Second screen 